\title{
POSITIONING ALGORITHMS FOR COOPERATIVE NETWORKS IN THE PRESENCE OF AN UNKNOWN TURN-AROUND TIME
}

\author{
Mohammad Reza Gholami ${ }^{\dagger}$ Sinan Gezici ${ }^{\sharp}$, Erik G. Ström ${ }^{\dagger}$, and Mats Rydström ${ }^{\dagger}$ \\ $\dagger$ Chalmers University of Technology, Department of Signals and Systems, Gothenburg, Sweden \\ $\sharp$ Bilkent University, Department of Electrical and Electronics Engineering, Ankara, Turkey
}

\begin{abstract}
This paper addresses the problem of single node positioning in cooperative network using hybrid two-way time-of-arrival and timedifference-of-arrival where, the turn-around time at the target node is unknown. Considering the turn-around time as a nuisance parameter, the derived maximum likelihood estimator (MLE) brings a difficult global optimization problem due to local minima in the cost function of the MLE. To avoid drawbacks in solving the MLE, we obtain a linear two-step estimator using non-linear pre-processing which is algebraic and closed-form in each step. To compare different methods, Cramér-Rao lower bound (CRLB) is derived. Simulation results confirm that the proposed linear estimator attains the CRLB for sufficiently high signal-to-noise ratios.
\end{abstract}

Index Terms - Cooperative positioning, linear estimator, and wireless sensor networks.

\section{INTRODUCTION}

Positioning algorithms based on TOA (or TDOA) need a synchronized network [1] that can be handled using different synchronization techniques $[2,3]$. The process of synchronizing the sensor nodes is a cumbersome and costly task. Alternatively, two-way time-ofarrival (TW-TOA) has been considered as an effective approach in the positioning literature (e.g., [4]), mainly due to its accuracy and lack of synchronization requirements. In this approach, a reference node sends a signal to a target node, and waits for a response from it. The round-trip time delay between the reference node and the target node gives an estimate of the distance between them.

As the number of reference nodes in a wireless sensor network (WSN) increases, the position of the target node can be estimated more accurately via TW-TOA estimation. Since, in practice, there are some limitations on increasing the number of reference nodes due to power and complexity constraints [5], the idea of cooperation between reference nodes is proposed in [6] to decrease the number of transmissions, and its theoretical analysis is presented in [4]. In this method, some reference nodes, called primary reference nodes (PRNs), initiate position estimation by sending a signal to a target. The target replies to received signals by sending an acknowledgement. Suppose that there are some other reference nodes, which can listen to both signals, and are called as secondary reference nodes (SRNs). It has been shown that the SRNs can help the PRNs to estimate the target position more accurately [4].

In positioning literature, it is commonly assumed that either an estimate of the turn-around time is available [4] or it is extremely

This work was supported in part by the European Commission in the framework of the FP7 Network of Excellence in Wireless COMmunication NEWCOM++ (contract no. 216715) and in part by the Swedish Research Council (contract no. 2007-6363) small $[7,8]$. On the contrary, in current work, we assume that no a-priori knowledge about the turn-around time is available and we just assume that it is a fixed unknown value for all links. In fact, we model it as a nuisance parameter that can be estimated jointly with the position of the target node. The maximum likelihood estimator (MLE) derived for this problem results in a difficult global optimization problem due to local minima in the MLE objective function. To cope the difficulty in solving the MLE for this problem, we first linearize measurements and obtain a linear model based on unknown parameters, i.e., target's position and turn-around time, and then a linear estimator is extracted. To improve the performance of the linear estimator we take the relation between estimation parameters in the first step into account and obtain a refining step. In order to evaluate the performance of the proposed method, the Cramér-Rao lower bound (CRLB) is obtained for this problem. Simulation results show that for sufficiently large SNRs, the proposed estimator attains the CRLB.

In summary, the main contributions of this paper are introducing the idea of joint estimation of the turn-around time and the position and proposing a two-step linear estimator as well as deriving the MLE and the CLRB.

The remainder of the paper is organized as follows. Section 2 explains the signal model considered in this paper. The MLE and CRLB are derived in Section 3.1 and Section 3.2, respectively. The two-step linear estimator is obtained in Section 4. Simulation results are discussed in Section 5. Finally Section 6 makes some concluding remarks.

\section{SIGNAL MODEL}

Let us consider a two-dimensional network with $N+M$ reference nodes located at known positions, $\mathbf{a}_{i}=\left[\begin{array}{ll}a_{i 1} & a_{i 2}\end{array}\right]^{T} \in \mathbb{R}^{2}, i=$ $1, \ldots, N+M$. Suppose that the first $N$ sensors, as PRNs, are used to measure the TW-TOA between them and the target to be located and that $M$ SRNs are able to listen and measure signals transmitted by the PRNs and the target. Let $\mathcal{C}=\{(i, j) \mid \mathrm{PRN} i$ and SRN $j$ are connected $\}$ denote the set of all pairs with one primary node and one secondary node that are connected. The TW-TOA measurement between primary node $i$ and target, located at coordinates $\boldsymbol{\theta}=\left[\begin{array}{ll}x_{1} & x_{2}\end{array}\right]^{T} \in \mathbb{R}^{2}$, can be written as [4]

$$
\hat{t}_{i}=\frac{r_{i}}{c}+\frac{T_{i}^{\mathrm{ar}}}{2}+\frac{\tilde{n}_{T, i}}{2}+\frac{\tilde{n}_{i, T}}{2}, i=1, \ldots, N,
$$

where $c$ is the speed of propagation, $r_{i}=\left\|\mathbf{a}_{i}-\boldsymbol{\theta}\right\|$ is the Euclidean distance between the $i$ th PRN and the point $\boldsymbol{\theta}, T_{i}^{\mathrm{ar}}$ is the turn-around time at the target node, $\tilde{n}_{i, T}$ is the TOA estimation error at the target node for the signal transmitted by the $i$ th PRN, and $\tilde{n}_{T, i}$ is the TOA estimation at the $i$ th PRN for the signal transmitted from the target 


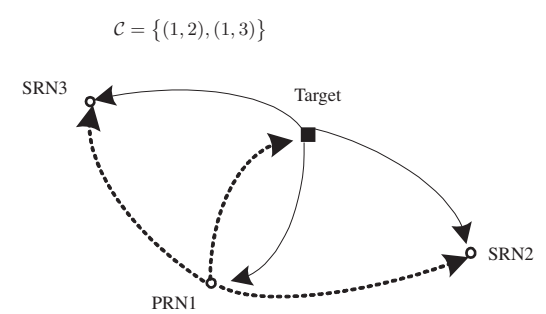

(a)

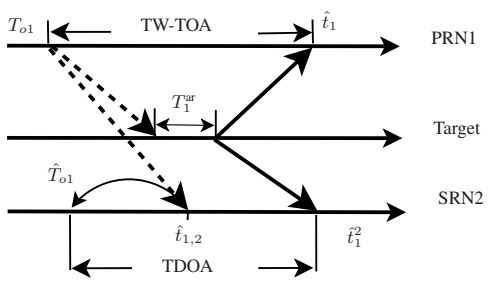

(b)

Fig. 1. (a) A cooperative network consists of one PRN and two SRNs (b) PRN1 sends its signal at $T_{o_{1}}$ and target node replies the received signal after $T_{1}^{\mathrm{ar}}$. Both signals are received in SNR2.

node. The estimation errors are modeled as $\tilde{n}_{T, i} \sim \mathcal{N}\left(0, \sigma_{T, i}^{2} / c^{2}\right)$ and $\tilde{n}_{i, T} \sim \mathcal{N}\left(0, \sigma_{i, T}^{2} / c^{2}\right)$ [4], where $\mathcal{N}\left(x_{1}, x_{2}\right)$ represents a Gaussian random variable (vector) with mean $x_{1}$ and variance (covariance matrix) $x_{2}$. The TOA estimate of the received signal from the $i$ th $\mathrm{PRN}$ in the $j$ th SRN is

$$
\hat{t}_{i, j}=T_{o_{i}}+\frac{r_{i, j}}{c}+\tilde{n}_{i, j},(i, j) \in \mathcal{C},
$$

where the $i$ th PRN sends its signal at time instant $T_{o_{i}}$, that is unknown to the $j$ th SRN, $r_{i, j}=\left\|\mathbf{a}_{i}-\mathbf{a}_{j}\right\|$ is the distance between the $i$ th PRN and the $j$ th SRNs, and $\tilde{n}_{i, j}$ is modeled as $\tilde{n}_{i, j} \sim \mathcal{N}\left(0, \sigma_{i, j}^{2} / c^{2}\right)$. Suppose that the response signal from the target to this signal is also received by the $j$ th SRN. The TOA estimate for this signal is

$$
\hat{t}_{i}^{j}=T_{o_{i}}+\frac{r_{i}}{c}+\frac{r_{j}}{c}+T_{i}^{\mathrm{ar}}+\tilde{n}_{i, T}+\tilde{n}_{T, j}, \quad(i, j) \in \mathcal{C} .
$$

Let us consider Fig. 1(a) where the PRN1 sends a signal to the target and the target replies to this signal after $T_{1}^{\text {ar }}$ (see Fig. 1(b)) Suppose that two other nodes (SRN2 and SRN3) listen to both signals. Since the distances between the reference nodes are known, it is possible in the secondary node to estimate the time reference $T_{o_{1}}$ from (2) (see Fig. 1(b)); Hence, the SRNs are able to estimate the overall distance from the PRN to the target and the target to the SRN plus the additional distance due to the delay $T_{i}^{\mathrm{ar}}$ as follows

$$
\begin{aligned}
& z_{i}^{j}=c\left(\hat{t}_{i}^{j}-\hat{T}_{o_{i}}\right)=r_{i}+r_{j}+c T_{i}^{\mathrm{ar}}+n_{i, T}+n_{T, j}-n_{i, j}, \\
& (i, j) \in \mathcal{C},
\end{aligned}
$$

where $n_{i, T}=c \tilde{n}_{i, T}, n_{T, j}=c \tilde{n}_{T, j}, n_{i, j}=c \tilde{n}_{i, j}$, and $\hat{T}_{o_{i}}$ is an estimate of $T_{o_{i}}$ (Fig. 1(b)), e.g., $\hat{T}_{o_{i}}=\hat{t}_{i, j}-r_{i, j} / c=T_{o_{i}}+\tilde{n}_{i, j}$. From (1), the distance estimate to the target in the $i$ th PRN plus additional distance due to $T_{i}^{\mathrm{ar}}$ is expressed as

$$
z_{i}=c \hat{t}_{i}=r_{i}+c \frac{T_{i}^{\mathrm{ar}}}{2}+\frac{n_{i, T}}{2}+\frac{n_{T, i}}{2}, i=1, \ldots, N
$$

where $n_{i, T}=c \tilde{n}_{i, T}$ and $n_{T, i}=c \tilde{n}_{T, i}$.

Since the turn-around time depends on the processing time at the target node, it is then reasonable to assume a constant value for all links; that is $T_{i}^{\mathrm{ar}}=T^{\mathrm{ar}}$.

\section{OPTIMAL ESTIMATOR AND THEORETICAL LIMITS}

\subsection{Maximum likelihood estimator}

Assuming a fully connected network, let the vector of measurements $\mathbf{z}$ be expressed as,

$$
\mathbf{z}=\left[z_{1} \ldots z_{N} z_{1}^{1} \ldots z_{1}^{M} \ldots z_{N}^{1} \ldots z_{N}^{M}\right]^{T} .
$$

It is clear that the vector $\mathbf{z}$ can be modeled as a Gaussian random vector $\mathbf{z} \sim \mathcal{N}(\boldsymbol{\mu}, \mathbf{C})$, where mean $\boldsymbol{\mu}=\left[\begin{array}{lll}\mu_{1} & \ldots \mu_{N} & \mu_{1}^{1} \ldots \mu_{1}^{M} \ldots\end{array}\right.$ $\left.\mu_{N}^{1} \ldots \mu_{N}^{M}\right]^{T}$ and covariance matrix $\mathbf{C}$ are,

$$
\begin{aligned}
& \mu_{i}=r_{i}+c \frac{T^{\mathrm{ar}}}{2}, \quad \mu_{i}^{j}=r_{i}+r_{N+j}+c T^{\mathrm{ar}}, \\
& \mathbf{C}=\mathbb{E}\left\{(\mathbf{z}-\boldsymbol{\mu})(\mathbf{z}-\boldsymbol{\mu})^{T}\right\}=\left[\begin{array}{ll}
\mathbf{C}_{11} & \mathbf{C}_{12} \\
\mathbf{C}_{21} & \mathbf{C}_{22}
\end{array}\right],
\end{aligned}
$$

where $\mathbb{E}$ denotes the expectation operator and matrices $\mathbf{C}_{11} \in \mathbb{R}^{N \times N}$ $\mathbf{C}_{12}=\mathbf{C}_{21}^{T} \in \mathbb{R}^{N \times N M}$, and $\mathbf{C}_{22} \in \mathbb{R}^{N M \times N M}$ can be obtained as follows:

$$
\begin{aligned}
& \mathbf{C}_{11}=\frac{1}{4} \operatorname{diag}\left(\left(\sigma_{T, 1}^{2}+\sigma_{1, T}^{2}\right), \ldots,\left(\sigma_{T, N}^{2}+\sigma_{N, T}^{2}\right)\right), \\
& \mathbf{C}_{12}=\left[\begin{array}{ccc}
\mathbf{v}_{1}^{T} & \ldots & \mathbf{0} \\
\vdots & \ddots & \vdots \\
\mathbf{0} & \ldots & \mathbf{v}_{N}^{T}
\end{array}\right], \mathbf{v}_{i}=\frac{\sigma_{T, i}^{2}}{2} \mathbf{1}_{M}, \mathbf{1}_{M}=[1 \ldots 1]^{T} \\
& \mathbf{C}_{22}=\operatorname{blkdiag}\left(\mathbf{W}_{1}, \mathbf{W}_{2}, \ldots, \mathbf{W}_{N}\right), \quad \mathbf{W}_{i}=\sigma_{T, i}^{2} \mathbf{1}_{M} \mathbf{1}_{M}^{T} \\
& +\operatorname{diag}\left(\sigma_{T, N+1}^{2}+\sigma_{i, N+1}^{2}, \ldots, \sigma_{T, N+M}^{2}+\sigma_{i, N+M}^{2}\right),
\end{aligned}
$$

where (blk) $\operatorname{diag}\left(X_{1}, \ldots, X_{N}\right)$ is a (block) diagonal matrix with diagonal element $X_{1}, \ldots, X_{N}$. The MLE is obtained by the following optimization problem $[9$, Ch. 7$]$

$$
\hat{\boldsymbol{\theta}}=\underset{\left[\boldsymbol{\theta} T^{\mathrm{ar}}\right] \in \mathbb{R}^{3}}{\arg \min }(\mathbf{z}-\boldsymbol{\mu})^{T} \mathbf{C}^{-1}(\mathbf{z}-\boldsymbol{\mu}) .
$$

With some manipulations, (8) can be expressed as

$$
\begin{aligned}
& \hat{\boldsymbol{\theta}}=\underset{\left[\boldsymbol{\theta} T^{\mathrm{ar}}\right] \in \mathbb{R}^{3}}{\arg \min } \sum_{i=1}^{N}\left\{\left(\frac{2}{\sigma_{i, T}^{2}}-\frac{1}{s_{i} \sigma_{i, T}^{4}}\right) \alpha_{i}^{2}\right. \\
& -\frac{1}{s_{i}}\left(\sum_{j=N+1}^{M+N} \frac{\alpha_{i, j}}{2\left(\sigma_{T, j}^{2}+\sigma_{i, j}^{2}\right)}\right)^{2}+\sum_{j=N+1}^{N+M} \frac{\alpha_{i, j}^{2}}{2\left(\sigma_{T, j}^{2}+\sigma_{i, j}^{2}\right)} \\
& \left.-\frac{\alpha_{i}}{s_{i} \sigma_{i, T}^{2}} \sum_{j=N+1}^{N+M} \frac{\alpha_{i, j}}{\left(\sigma_{T, j}^{2}+\sigma_{i, j}^{2}\right)}\right\},
\end{aligned}
$$

where

$$
\begin{aligned}
& s_{i}=\frac{1}{2 \sigma_{T, i}^{2}}+\frac{1}{2 \sigma_{i, T}^{2}}+\sum_{j=N+1}^{M+N} \frac{1}{2\left(\sigma_{T, j}^{2}+\sigma_{i, j}^{2}\right)}, \\
& \alpha_{i}=z_{i}-r_{i}-\frac{1}{2} c T^{\mathrm{ar}}, \quad \alpha_{i, j}=z_{i}^{j}-r_{i}-r_{j}-c T^{\mathrm{ar}} .
\end{aligned}
$$

As can be seen the MLE brings a difficult global optimization problem due to non-linearity and non-convexity issues. 


\subsection{Cramér-Rao lower bound}

Considering the measurement vector (6) with mean $\boldsymbol{\mu}$ and covariance matrix $\mathbf{C}$, i.e., (7), the Fisher information matrix can be computed as [9, Ch. 3] $[I]_{n m}=\left[\frac{\partial \boldsymbol{\mu}}{\partial \psi_{n}}\right]^{T} \mathbf{C}^{-1}\left[\frac{\partial \boldsymbol{\mu}}{\partial \psi_{m}}\right], n=1,2,3$, $m=1,2,3$.

Simple calculations considering $\boldsymbol{\psi}=\left[\begin{array}{lll}x & y & c T^{\mathrm{ar}}\end{array}\right]^{T}$ yeild

$$
\begin{aligned}
& {\left[\frac{\partial \boldsymbol{\mu}}{\partial \psi_{n}}\right]^{T}=\left[\frac{\partial \mu_{1}}{\partial \psi_{n}} \ldots \frac{\partial \mu_{N}}{\partial \psi_{n}} \ldots \frac{\partial \mu_{N}^{1}}{\partial \psi_{n}} \ldots \frac{\partial \mu_{N}^{M}}{\partial \psi_{n}}\right],} \\
& \frac{\partial \mu_{i}}{\partial \psi_{1}}=\frac{x_{1}-x_{i, 1}}{r_{i}}, \frac{\partial \mu_{i}^{j}}{\partial \psi_{1}}=\frac{x_{1}-x_{i, 1}}{r_{i}}+\frac{x_{1}-x_{N+j, 1}}{r_{N+j}}, \\
& \frac{\partial \mu_{i}}{\partial \psi_{2}}=\frac{x_{2}-x_{i, 2}}{r_{i}}, \frac{\partial \mu_{i}^{j}}{\partial \psi_{2}}=\frac{x_{2}-x_{i, 2}}{r_{i}}+\frac{x_{2}-x_{N+j, 2}}{r_{N+j}}, \\
& \frac{\partial \mu_{i}}{\partial \psi_{3}}=\frac{1}{2}, \frac{\partial \mu_{i}^{j}}{\partial \psi_{3}}=1 .
\end{aligned}
$$

The lower bound on any unbiased estimator is then given by

$$
\begin{aligned}
& \mathbb{E}\left\{\|\hat{\boldsymbol{\theta}}-\boldsymbol{\theta}\|^{2}\right\} \\
& \geq \frac{I_{33}\left(I_{22}+I_{11}\right)-\left(I_{32}^{2}+I_{13}^{2}\right)}{I_{33}\left(I_{11} I_{22}-I_{12}^{2}\right)+\left(2 I_{31} I_{23} I_{12}-I_{22} I_{13}^{2}-I_{11} I_{23}^{2}\right)} \\
& =\frac{I_{33}-\left(I_{32}^{2}+I_{13}^{2}\right)\left(I_{22}+I_{11}\right)^{-1}}{I_{33}(\Upsilon)+\left(2 I_{31} I_{23} I_{12}-I_{22} I_{13}^{2}-I_{11} I_{23}^{2}\right)\left(I_{22}+I_{11}\right)^{-1}}
\end{aligned}
$$

where $1 / \Upsilon=\left(I_{22}+I_{11}\right)\left(I_{11} I_{22}-I_{12}^{2}\right)^{-1}$ is the lower bound of any unbiased estimator when the perfect knowledge of the turn-around time is available [4]. Note that the whole results obtained here and previous section can be applied to the conventional network where there are only primary nodes.

\section{LINEAR ESTIMATOR}

Suppose that the level of noise is small. For the $i$ th PRN, moving the term $1 / 2 c T_{i}^{\mathrm{ar}}$ in (5) to the left-hand side and then squaring both sides yields, after dropping the small term and recalling $T_{i}^{\mathrm{ar}}=T^{\mathrm{ar}}$,

$$
\begin{aligned}
& z_{i}^{2}-c z_{i} T^{\mathrm{ar}}+\frac{1}{4} c^{2}\left(T^{\mathrm{ar}}\right)^{2} \\
& \approx\|\boldsymbol{\theta}\|^{2}-2 \mathbf{a}_{i}^{T} \boldsymbol{\theta}+\left\|\mathbf{a}_{i}\right\|^{2}+2 r_{i} \vartheta_{i}, i=1, \ldots, N,
\end{aligned}
$$

where $\vartheta_{i}=1 / 2 n_{i, T}+1 / 2 n_{T, i}$.

Eq. (11) can be written as

$$
\tilde{z}_{i}=z_{i}^{2}-\left\|\mathbf{a}_{i}\right\|^{2}=\left[-2 \mathbf{a}_{i}^{T} z_{i} 1\right] \psi+2 r_{i} \vartheta_{i}, i=1, \ldots, N
$$

where $\boldsymbol{\psi}=\left[\begin{array}{ll}\boldsymbol{\theta}^{T} & c T^{\mathrm{ar}}\|\boldsymbol{\theta}\|^{2}-\left(1 / 2 c T^{\mathrm{ar}}\right)^{2}\end{array}\right]^{T}$.

For the time-difference-of-arrival (TDOA) measurement in the $j$ th SRN, i.e., (4), let us first arrange a new set of measurements, subtract (4) from (5), as follows

$$
\tilde{z}_{i}^{j}=z_{i}^{j}-z_{i}=r_{j}+\frac{1}{2} c T^{\mathrm{ar}}+\epsilon_{i}^{j},(i, j) \in \mathcal{C},
$$

where $\epsilon_{i}^{j}=n_{T, j}+1 / 2 n_{i, T}-1 / 2 n_{T, i}-n_{i, j}$. Now similar to (11), we can linearize (12) to get, assuming small noise $\epsilon_{i}^{j}$,

$$
\begin{aligned}
& \left(\tilde{z}_{i}^{j}\right)^{2}-c \tilde{z}_{i}^{j} T^{\mathrm{ar}}+\frac{1}{4} c^{2}\left(T^{\mathrm{ar}}\right)^{2} \\
& \approx\|\boldsymbol{\theta}\|^{2}-2 \mathbf{a}_{j}^{T} \boldsymbol{\theta}+\left\|\mathbf{a}_{j}\right\|^{2}+2 r_{j} \epsilon_{i}^{j}, \quad(i, j) \in \mathcal{C} .
\end{aligned}
$$

Therefore a linear model for measurement in $\operatorname{SRN} j$ is obtained as follows

$$
\overline{\tilde{r}}_{i}^{j}=\left(\tilde{z}_{i}^{j}\right)^{2}-\left\|\mathbf{a}_{j}\right\|^{2}=\left[-2 \mathbf{a}_{j}^{T} \tilde{z}_{i}^{j} 1\right] \boldsymbol{\psi}+2 r_{j} \epsilon_{i}^{j},(i, j) \in \mathcal{C} .
$$

The linear set of equations can be written as

$$
\mathbf{d}=\mathbf{A} \psi+\nu,
$$

where vectors $\mathbf{d}, \boldsymbol{\nu}$, and matrix $\mathbf{A}$ are obtained as follows

$$
\begin{aligned}
& \mathbf{d}=\left[\begin{array}{lllll}
\tilde{z}_{1} & \ldots & \tilde{z}_{N} \ldots & \overline{\tilde{r}}_{N}^{1} \ldots & \overline{\tilde{r}}_{N}^{M}
\end{array}\right]^{T}, \mathbf{A}=\left[\begin{array}{llll}
\mathbf{A}_{1}^{T} & \mathbf{B}_{1}^{T} & \ldots & \mathbf{B}_{N}^{T}
\end{array}\right]^{T},
\end{aligned}
$$

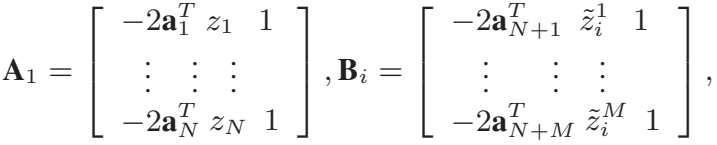

$$
\begin{aligned}
& \boldsymbol{\nu}=2\left[r_{1} \vartheta_{1} \ldots r_{N} \vartheta_{N} \ldots r_{N+1} \epsilon_{N}^{N+1} \ldots r_{N+M} \epsilon_{N}^{N+M}\right]^{T}
\end{aligned}
$$

Using the least squares criterion [9, Ch. 8], a closed-form solution for (14) can be obtained as

$$
\hat{\boldsymbol{\psi}}=\left(\mathbf{A}^{T} \mathbf{C}_{\boldsymbol{\nu}}^{-1} \mathbf{A}\right)^{-1} \mathbf{A}^{T} \mathbf{C}_{\boldsymbol{\nu}}^{-1} \mathbf{d} .
$$

When matrix $\mathbf{A}$ is ill-conditioned (this case sometimes happens to a medium or large scale network, e.g., network considered in simulation part) and then we can use the regularization technique [10, Ch 6] to get

$$
\hat{\boldsymbol{\psi}}=\left(\mathbf{A}^{T} \mathbf{C}_{\boldsymbol{\nu}}^{-1} \mathbf{A}+\lambda \mathbf{I}_{4}\right)^{-1} \mathbf{A}^{T} \mathbf{C}_{\boldsymbol{\nu}}^{-1} \mathbf{d},
$$

where parameter $\lambda$ defines the trade off between $\|\mathbf{d}-\mathbf{A} \boldsymbol{\psi}\|^{2}$ and $\|\boldsymbol{\psi}\|^{2}, \mathbf{I}_{M}$ is the $M \times M$ identity matrix, and the covariance matrix $\mathbf{C}_{\nu}$ of noise vector $\boldsymbol{\nu}$ is computed as follows

$$
\mathbf{C}_{\boldsymbol{\nu}}=\mathbb{E}\left\{(\boldsymbol{\nu}-\mathbb{E}\{\boldsymbol{\nu}\})(\boldsymbol{\nu}-\mathbb{E}\{\boldsymbol{\nu}\})^{T}\right\}=\left[\begin{array}{ll}
\mathbf{C}_{\boldsymbol{\nu}_{11}} & \mathbf{C}_{\boldsymbol{\nu}_{12}} \\
\mathbf{C}_{\boldsymbol{\nu}_{21}} & \mathbf{C}_{\boldsymbol{\nu}_{22}}
\end{array}\right],
$$

where matrices $\mathbf{C}_{\boldsymbol{\nu}_{11}} \in \mathbb{R}^{N \times N}, \mathbf{C}_{\boldsymbol{\nu}_{12}}=\mathbf{C}_{\boldsymbol{\nu}_{21}}^{T} \in \mathbb{R}^{N \times N M}$, and $\mathbf{C}_{\boldsymbol{\nu}_{22}} \in \mathbb{R}^{N M \times N M}$ are given by

$$
\begin{aligned}
& \mathbf{C}_{\boldsymbol{\nu}_{11}}=\operatorname{diag}\left(r_{1}^{2}\left(\sigma_{T, 1}^{2}+\sigma_{1, T}^{2}\right), \ldots, r_{N}^{2}\left(\sigma_{T, N}^{2}+\sigma_{N, T}^{2}\right)\right), \\
& \mathbf{C}_{\boldsymbol{\nu}_{12}}=\left[\begin{array}{ccc}
\mathbf{r}_{1}^{T} & \ldots & \mathbf{0} \\
\vdots & \ddots & \vdots \\
\mathbf{0} & \ldots & \mathbf{r}_{N}^{T}
\end{array}\right], \mathbf{C}_{\boldsymbol{\nu}_{22}}=\operatorname{blkdiag}\left(\mathbf{R}_{1}, \ldots, \mathbf{R}_{N}\right), \\
& \mathbf{r}_{i}=r_{i}\left[r_{N+i}\left(\sigma_{T, i}^{2}+\sigma_{i, T}^{2}\right) \ldots r_{N+M}\left(\sigma_{T, i}^{2}+\sigma_{i, T}^{2}\right)\right]^{T}, \\
& \mathbf{R}_{i}=\left(\sigma_{T, i}^{2}+\sigma_{i, T}^{2}\right)\left[\begin{array}{c}
r_{N+1} \\
\vdots \\
r_{N+i}
\end{array}\right]\left[r_{N+1} \ldots r_{N+M}\right]+4 \operatorname{diag}( \\
& \left.r_{N+1}^{2}\left(\sigma_{N+1, T}^{2}+\sigma_{T, N+1}^{2}\right) \ldots r_{N+M}^{2}\left(\sigma_{T, N+M}^{2}+\sigma_{N+M, T}^{2}\right)\right) .
\end{aligned}
$$

The covariance matrix of $\hat{\psi}$ can be computed as

$$
\operatorname{cov}(\hat{\boldsymbol{\psi}})=\left(\mathbf{A}^{T} \mathbf{C}_{\boldsymbol{\nu}}^{-1} \mathbf{A}+\lambda \mathbf{I}_{4}\right)^{-1} \mathbf{A}^{T} \mathbf{C}_{\boldsymbol{\nu}}^{-1} \mathbf{A}^{T}\left(\mathbf{A}^{T} \mathbf{C}_{\boldsymbol{\nu}}^{-1} \mathbf{A}+\lambda \mathbf{I}_{4}\right)^{-1} .
$$

To compute the covariance matrix $\mathbf{C}_{\nu}$ instead of the real distances between reference nodes to the target, the estimated distances can be used [11]. To improve the first step estimator, we can take the relation between elements of $\hat{\psi}$ in (16) into account and obtain a 
refining step. Suppose that each element of (16) can be written as

$$
\begin{aligned}
& {[\hat{\boldsymbol{\psi}}]_{1}=x_{1}+e_{1},[\hat{\boldsymbol{\psi}}]_{2}=x_{2}+e_{2},[\hat{\boldsymbol{\psi}}]_{3}=c T^{\mathrm{ar}}+e_{3},} \\
& {[\hat{\boldsymbol{\psi}}]_{4}=\|\boldsymbol{\theta}\|^{2}-\frac{1}{4}\left(c T^{\mathrm{ar}}\right)^{2}+e_{4},}
\end{aligned}
$$

where $\boldsymbol{\epsilon}=\left[\begin{array}{llll}e_{1} & e_{2} & e_{3} & e_{4}\end{array}\right]^{T}$ is the error of estimation. Let the errors of estimation be considerably small. Therefore squaring the both sides of the first three elements of (17) yields, after dropping the small terms,

$$
\begin{aligned}
& {[\hat{\boldsymbol{\psi}}]_{1}^{2} \simeq x_{1}^{2}+2 x_{1} e_{1}, \quad[\hat{\boldsymbol{\psi}}]_{2}^{2} \simeq x_{2}^{2}+2 x_{2} e_{2},} \\
& {[\hat{\boldsymbol{\psi}}]_{3}^{2} \simeq\left(c T^{\mathrm{ar}}\right)^{2}+2 c T^{\mathrm{ar}} e_{3} .}
\end{aligned}
$$

From (17) and (18), we can write

$$
\mathbf{b}=\mathbf{B} \phi+\zeta,
$$

where the parameters $\mathbf{b}, \mathbf{B}, \phi$, and $\zeta$ are computed as follows

$$
\begin{aligned}
& \mathbf{b}=\left[[\hat{\boldsymbol{\psi}}]_{1}^{2}[\hat{\boldsymbol{\psi}}]_{2}^{2}[\hat{\boldsymbol{\psi}}]_{3}^{2}[\hat{\boldsymbol{\psi}}]_{4}\right]^{T}, \mathbf{B}=\left[\begin{array}{ccc}
1 & 0 & 0 \\
0 & 1 & 0 \\
0 & 0 & 1 \\
1 & 1 & -\frac{1}{4}
\end{array}\right], \\
& \boldsymbol{\phi}=\left[x_{1}^{2} x_{2}^{2}\left(c T^{\mathrm{ar}}\right)^{2}\right]^{T}, \boldsymbol{\zeta}=\left[2 x_{1} e_{1} 2 x_{2} e_{2} 2 c T^{a r} e_{3} e_{4}\right]^{T} .
\end{aligned}
$$

The least squares approximation of (19) is obtained as

$$
\hat{\phi}=\left(\mathbf{B}^{T} \mathbf{C}_{\zeta}^{-1} \mathbf{B}\right)^{-1} \mathbf{B}^{T} \mathbf{C}_{\boldsymbol{\zeta}}^{-1} \mathbf{b},
$$

where covariance matrix $\mathbf{C}_{\zeta}^{-1}$ can be computed as [11]

$$
\mathbf{C}_{\boldsymbol{\zeta}}=\mathbb{E}\left\{(\mathbf{b}-\mathbf{B} \phi)(\mathbf{b}-\mathbf{B} \phi)^{T}\right\}=\boldsymbol{\Lambda} \operatorname{cov}(\hat{\boldsymbol{\psi}}) \boldsymbol{\Lambda}
$$

where $\boldsymbol{\Lambda}=\operatorname{diag}\left(2 x_{1}, 2 x_{2}, 2 c T^{\mathrm{ar}}, 1\right)$. To compute matrix $\boldsymbol{\Lambda}$, the estimated parameters from (16) are used. Finally the target position can be obtained as follows

$$
\hat{x}_{1}=\frac{\left|[\hat{\boldsymbol{\psi}}]_{1}\right|}{[\hat{\boldsymbol{\psi}}]_{1}} \sqrt{\left|[\hat{\boldsymbol{\phi}}]_{1}\right|}, \hat{x}_{2}=\frac{\left|[\hat{\boldsymbol{\psi}}]_{2}\right|}{[\hat{\boldsymbol{\psi}}]_{2}} \sqrt{\left|[\hat{\boldsymbol{\phi}}]_{2}\right|} .
$$

The covariance matrix of the fine estimator in (22) can be computed similar to [11] as follows. Suppose the estimate in (20) can be written as

$$
\hat{\phi}_{\ell}=\phi_{\ell}+\tilde{\zeta}
$$

where $\tilde{\zeta}=\left[\begin{array}{lll}\tilde{\zeta}_{1} & \tilde{\zeta}_{2}\end{array}\right]$ is the error of estimation in (20). Using the firstorder Taylor series expression, assuming small error $\tilde{\zeta}$, we get

$$
\tilde{x}_{j}=\frac{\left|[\hat{\boldsymbol{\psi}}]_{j}\right|}{[\hat{\boldsymbol{\psi}}]_{j}}\left(\left|x_{j}\right|+\frac{1}{2\left|x_{j}\right|} \tilde{\zeta}_{j}\right), j=1,2
$$

Hence, the covariance matrix of $\tilde{\mathbf{x}}$ can be computed as

$$
\operatorname{cov}(\tilde{\mathbf{x}})=\tilde{\mathbf{B}}[\operatorname{cov}(\hat{\phi})]_{(1: 2,1: 2))} \tilde{\mathbf{B}}
$$

where $\tilde{\mathbf{B}}=\frac{1}{2} \operatorname{diag}\left(\left|x_{1}\right|^{-1},\left|x_{2}\right|^{-1}\right)$ and $[\mathbf{Z}]_{(1: n, 1: m)}$ denotes the upper left $n \times m$ part of matrix $\mathbf{Z}$.

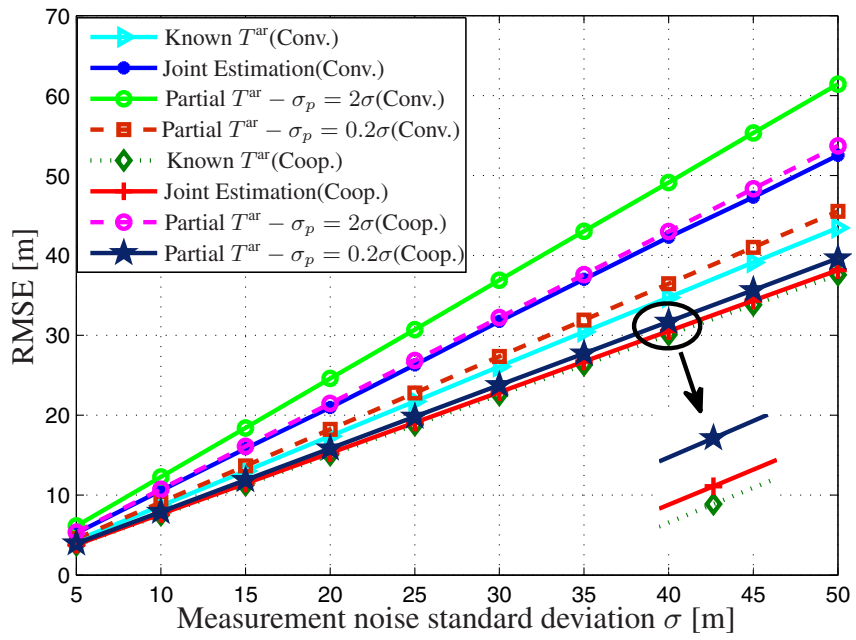

Fig. 2. RMSE of different CRLBs.

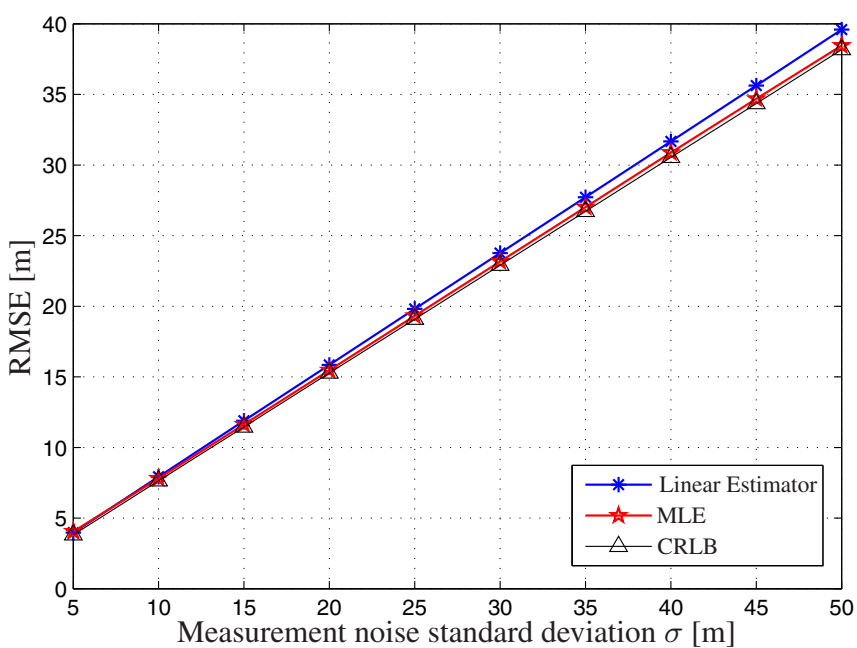

Fig. 3. RMSE of the CRLB, MLE, and linear estimators in cooperative cases.

\section{SIMULATION RESULTS}

In the simulation three PRNs and one SRN are located at the corner of a square area with coordinates $(-500 \mathrm{~m},-500 \mathrm{~m}),(-500 \mathrm{~m}$, $500 \mathrm{~m}),(500 \mathrm{~m}, 500 \mathrm{~m})$, and $(500 \mathrm{~m},-500 \mathrm{~m})$ respectively. A target is randomly placed inside the square area over a grid of $400 \mathrm{~m} \times$ $400 \mathrm{~m}$. We assume $\sigma_{T, i}=\sigma_{i, T}=\sigma_{i, j}=\sigma$. To study the effect of partial knowledge of the turn-around time, we model it as $T^{\mathrm{ar}} \sim \mathcal{N}\left(\mu_{T^{\mathrm{ar}}}, \sigma_{p}^{2}\right)$. In the simulation, we set $\sigma_{p}^{2}=2 \sigma^{2}$ and $\sigma_{p}^{2}=0.2 \sigma^{2}$, and we simply choose $\lambda=0.1$. The turn-around time is randomly drawn from $[0.11] \mu s$.

In Fig. 2 we plot the root-mean-square-error (RMSE) of the CRLB for different scenarios. To compute the CRLB, we get the average of the CRLBs for all realization of target and turn-around time. This figure shows that cooperation improves the accuracy of the estimation. It is also seen that for both cooperative and conventional networks joint estimation of turn-around time and position of the target deteriorates the accuracy of estimation compared to the case when perfect knowledge of the turn-around time is available. The inter- 


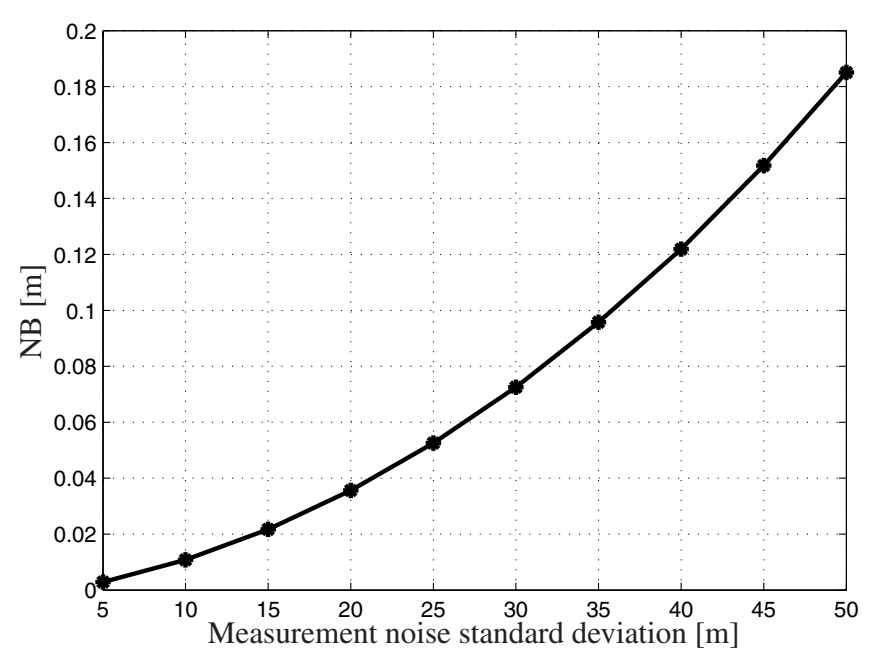

Fig. 4. Norm of bias (NB) for the linear estimator.

esting observation is that in cooperative case the difference between CRLB of position estimation with perfect knowledge of turn-around time and CRLB of joint position and turn around time estimation is very small compared to the non-cooperative case. Note that for the conventional network, just three PRNs are involved.

In the next simulation, we evaluate the performance of MLE and linear estimator for the cooperative network. Fig. 3 shows the RMSE of the linear estimator, MLE, and CRLB for cooperative network. As can be observed, the linear estimator attains the CRLB as well as MLE for high SNR.

To evaluate the bias of the estimator, we compute the norm of bias (NB) which we define as

$$
\mathrm{NB}=\|\mathrm{E}\{\hat{x}-x\}\| .
$$

We depicted the NB for linear estimator in Fig. 4. It shows that the absolute norm of bias increases with increasing the standard deviation of noise, but comparing NB with RMSE of the CLRB, we conclude that the proposed estimator can be considered as an unbiased estimator.

\section{CONCLUSION}

In this paper, we have studied the positioning problem in cooperative network using the hybrid two-way time of arrival and time difference of arrival in the presence of an unknown turn-around time at a target node. Considering the turn-around time as a nuisance parameter, the derived maximum likelihood estimator (MLE) is a difficult global optimization problem due to local minima in objective function. To avoid drawbacks in the MLE, we have used a linearization technique to obtain a linear estimator and subsequently applied a refining technique. The proposed estimator has a closed-form solution in each step and simulation results show that it is asymptotically efficient.

\section{REFERENCES}

[1] G. Mao and B. Fidan, Localization algorithms and strategies for wireless sensor networks. Information Science reference, Hershey. New York, 2009.

[2] Q. Chaudhari, E. Serpedin, and J. Kim, "Energy-efficient estimation of clock offset for inactive nodes in wireless sensor network," IEEE Trans. Inf. Theory, vol. 56, no. 1, pp. 582-596, Jan. 2010.

[3] I. Sari, E. Serpedin, K. Noh, Q. Chaudhari, and B. Suter, "On the joint synchronization of clock offset and skew in RBS-protocol," IEEE Trans. Commun., vol. 56, no. 5, pp. 700-703, May 2008.

[4] S. Gezici and Z. Sahinoglu, "Enhanced position estimation via node cooperation," in Proc. IEEE International Conference on Communications (ICC), Cape Town, South Africa, May 23-27, 2010.

[5] M. R. Gholami, "Positioning algorithms for wireless sensor networks," Licentiate thesis, Chalmers University of Technology, Mar. 2011.

[6] R. Fujiwara, K. Mizugaki, T. Nakagawa, D. Maeda, and M. Miyazaki, "TOA/TDOA hybrid relative positioning system using UWB-IR," in IEEE Radio and Wireless Week, Jan. 2009, pp. 679-682.

[7] M. R. Gholami, S. Gezici, E. G. Ström, and M. Rydström, "A distributed positioning algorithm for cooperative active and passive sensors," in Proc. IEEE International Symposium on Personal, Indoor and Mobile Radio Communications (PIMRC), Sep. 2010.

[8] — - "Hybrid TW-TOA/TDOA positioning algorithms for cooperative wireless networks," in Proc. IEEE International Conference on Communications (ICC) (to appear), Kyoto, Japan, Jun. 2011.

[9] S. M. Kay, Fundamentals of statistical signal processing: estimation theory. Upper Saddle River, NJ: Prentice-Hall, 1993.

[10] S. Boyd and L. Vandenberghe, Convex Optimization. Cambridge University Press, 2004.

[11] M. Sun and K. C. Ho, "Successive and asymptotically efficient localization of sensor nodes in closed-form," IEEE Trans. Signal Process., vol. 57, no. 11 , pp. 4522-4537, Nov. 2009. 